\title{
Non-violent Culture in Relation with Some Mental Disorders
}

\author{
Sareng Aziz Salih Iraq \\ Faculty of Language, Humanity School, Sociology department, Sulaimania University.
}

\begin{abstract}
The World Health Organization defined health in 1946 as a state of complete physical, mental and social well-being.

This definition underscores a very important point which is; health is as much a social as a biological issue, and it provides major clues as to how a society or nation works. Societies with a lot of sickness and early death are likely to be socially organized very differently from those where people live longer lives and experience less illness. Though there is some non-epidemic diseases like: suicide, the addiction of drugs, alcohol abuse and mental disorders, which lead to the exercise of violence in the society.

Epidemiology draws on the work of a wide variety of scientists and researchers, among them physicians, sociologists, demographers, biologist, anthropologists and psychologists.

These non-epidemic diseases need the knowledge how to take care of the mental-disorder, patient without violence, understanding the symptoms and give him/her the right treatment. There are some mental disorders, which are less identified in the third world. We see daily many children and adults with behavioral problems, such aggressive behavior. Many of the social and educational institutes like schools, families and places, face difficulties in the treatment of them. Often the only way to meet these situations is the violence.

And like sociologists we know that Violence breeds Violence.

Keywords: ADHD,Non-violence,Mental disorder
\end{abstract}

\section{Research problem}

Violence has been an issue for the Crime-and-Punish system for a long time, but in order to resist violence we have to resort to the social institutions. When a person with mental disorder, apply tohelp by a social institution, the important point is how -the disorder identifies of experts. The expert team usually, includes social workers, nurses, psychiatrists, psychologists, therapists, and personal who are working at the institutions. This team is working for the client's best. The case will be identified by the expert team after several meetings and conversations. They make a decision and a treatment plan. This decision is based on the quality and the quantity of knowledge by every member of the expert team. In undeveloped countries, the needed knowledge is concentrated by the head of the expert team only. And some mental disorders like ADHD, is still equaled with some psychological crises, when in fact. ADHD is genetics.

\section{Research importance}

This research is a theoretical study. The study will introduce the medical and labeling approaches to mental disorders. The study will put light on some less known mental disorders like ADHD. These disorders usually treated with violence, in most of the undeveloped countries. ADHD's prevalence worldwide is estimated to be a bit over $5 \%$ with most of the reported variability. $10 \%$ of males and (only) $4 \%$ of females have been diagnosed in the U.S.

\section{Researches aims}

This research will identify violence like a public health issue.

The aim of this research is dissemination of knowledge about some mental disorders like: ADHD, which means (Attention-deficit hyperactivity disorder).

The disorder typically presents itself during childhood, and is characterized by persistent patterns of inattention and/or hyperactivity, as well as forgetfulness, poor impulse control or impulsivity and distractibility.

\section{The first part}

Definition of the terms

Violence:

Violence is the exertion of physical force so as to injure or abuse, more frequently the word describes forceful human destruction of property or injury to persons, usually intentional, and forceful verbal and emotional abuse that harms others.

Non-violence: 
Whether held as a moral philosophy or only employed as an action strategy, rejects the use of physical or psychological violence in efforts to attain social, economic or political change.

Culture:

Generally refers to patterns of human activity and the symbolic structures that give such activities significance and importance. Different definitions of "culture" reflect different theoretical bases for understanding, or criteria for evaluating, human activity.

Relation: Is the connection between two or more subjects or persons?

\section{Mental disorders:}

Are terms used to refer a psychological or physiological pattern that occurs in an individual, is usually associated with distress or disability that is not expected as part of normal development or culture Categories of diagnoses in these schemes may include mood disorders, anxiety disorders, psychotic disorders, eating disorders, developmental disorder personality disorders, and many other categories.

\section{Mental disorders and violence}

The causes of mental disorders are complex, and interact and vary according to the particular disorder and individual. Genetics, early development, drugs, disease or injury. Neurocognitive and psychological mechanisms, and life experiences, society and culture can all contribute to the development or progression of different mental disorders.

the treatment of mental disorders may include the use of psychotherapy, psychiatric medication management, or other practices, in developed countries like USA and Europe. In other parts of world, especially in undeveloped countries, mental disorders seas like a fault of families. In these cu mental disorders covers on by the relatives and Friends. The treatment will be often psychiatric medication. The public fear of violence due to illness is a contentious topic. One US national survey indicated that a far higher percentage of Americans rated individuals described as displaying the characteristics of a mental disorder (for example

Schizophrenia.) as "likely to do something violent to others" compared to those described as being troubled'. Research indicates, on balance, a higher than average number of violent acts by some individuals with certain diagnoses, notably antisocial or psychopathic personality disorders, but conflicting findings about specific symptoms (for example between psychosis and violence in community settings) - but the mediating factors of such acts a most consistently found to be mainly socio-demographic and socio-economic factors such as being young, male, of lower socio-economic stage and, in particular, substance abuse (including alcohol). Findings consistently indicate that it is many times more likely that people diagnosed with a serious mental illness living in the community will be the victim rather than the perpetrator of violence. Violence by or against individuals with mental illness typically occurs in the context of complex social. Interactions including in atmosphere of mutually high 'expressed emotion", including within a family setting, as well as being an issue in healthcare set and the wider community.

Some theories of mental disorders in studying mental illness, we can draw on both medical model and a more sociological approach derived from labeling theory. Each model offers distinctive assumptions regarding treatment of people with mental disorders.

\section{The medical model}

This model views mental disorders as a disease derived from biological causes that can be treated through medical intervention. All mental dysfunctions are attributed to physical causes- whether physiological, biochemical, or genetic.

In the view of its critics, the medical model relies too heavily, on chemical interventions in creating people with mental disorders. Psychologist Albert Bandura (1969) argues that this approach has led to the "unremitting search for drugs as quick remedies for interpersonal problems, and long-term neglect of social variables as influential determinants of deviant response patterns.

\section{The labeling theory}

In contrast to the medical model, labeling theory, suggests that mental illness is not really an "illness" since the individual's problems arise from living in society and nor from physical maladies. psychiatrist Thomas Szasz (1974), in his book The myth of mental illness, which first appeared in 1961 advanced the view that numerous personality disorders are not "diseases" but simply patterns of conduct labeled as disorders by significant others. According to researcher's opinion:

In reviewing the medical and labeling models of mental illness, we can conclude that neither model offers a satisfactory overall explanation. 
Clearly, the medical model is persuasive because it pinpoints causes of mental illness and treatments for disorders. Yet proponents of the labeling perspective maintain that mental illness is a distinctively social process, whatever other processes are involved. From sociological perspective, the Ideal approach to mental illness integrates the insights of labeling theory with those of the medical approach.

\section{The Second Part}

What is ADHD Attention-Deficit Hyperactivity Disorder (ADHD), or Hyperkinetic Disorder as officially known in the UK though ADHD is more commonly used, is generally considered to be a developmental disorder, largely neurological in nature the disorder typically presents itself during childhood, and is characterized by a persistent pattern of inattention and/or hyperactivity, as well as forgetfulness, poor impulse control or impulsivity, and distractibility.

About $60 \%$ of children diagnosed with ADHD retain the disorder as adults. The disorder appears to be highly heritable, with contributions on occasion from trauma or toxic exposure. Methods of treatment usually involve some combination of medications, behavior modifications, life style changes, and counseling.

\section{History}

Some sources claim to have identified historical and literary references to ADHD before 1900 . However, the condition we refer to as "ADHD" dates to the mid-twentieth century, when physicians developed a diagnosis for a set of conditions variously referred to as "minimal brain damage".

If disabilities or hyperactivity in $493 \mathrm{BC}$, physician-scientist Hippocrates described a condition that seems to be compatible with what we now know as ADHD. He described patients who had "quickened responses to sensory experience, but also less tenaciousness because the soul moves on quickly to the next impression". Hippocrates attributed this condition to an "overbalance of fire over water.

\section{Diagnosis}

These following symptoms of inattention:

1.Often has trouble keeping attention on tasks

2- Often does not seem to listen of play activities

3- Often does not follow when spoken to directly instructions and fails to finish schoolwork, chores duties in the workplace (not due to oppositional

4- Behavior or failure to understand instruction

5- Often has trouble organizing activities dislikes, or doesn't want to do things that take a lot of mental effort for a long- period of time

6- Often loses things as schoolwork or homework needed for tasks and activities (e.g. toys, school)

7- Often forget assignments, pencils, books, or tool

8- Often forgetful in daily activities easily distracted

\section{Symptoms of hyperactivity}

1- Often fidgets with hands or feet or squirms?

2- Often gets up from seat when remaining in seat.

3- Often runs about or climbs when seat is expected and where it is not appropriate (adolescents or adults)

4- Often has trouble playing may feel very restless).

5 - Is often "on or

enjoying leisure activities quietly.

6-the go" or often acts as if "driven by a motto Often talks excessively.

\section{Symptoms of impulsiveness}

I- Often blurts out answers before questions.

2-Often has trouble waiting one's have been finished.

3- Often interrupts or intrudes on others (e.g. turn butts into conversations or games.

\section{Causes}

There is a wide body of evidence which indicates that ADHD is genetics.

\section{Prevention}

There is no known way to prevent ADHD.

Some studies indicate an association between mothers who smoke during pregnancy and a higher rate ADHD in their children.

\section{Treatment}


ADHD is treated most effectively with medication. Psychotherapy is another option, with without medication.

\section{Conclusion}

As we pointed out, these symptoms are very ordinary in the most social institutions like families and schools, in our society, but the only difference is that we did not give it the name ADHD. And following on, we did not give it the right treatment.

Often the parents, teachers, adults around the person with ADHD symptoms, treat him/her with violence. $\mathrm{He} / \mathrm{she}$ explains healthy but with some behavioral problems. Indeed, this person with ADHD has a developmental disorder that is often said to be Neurological in nature.

\section{References}

[1]. Society, J.Macionis, John, eihth dition,2006, by Pearson Education, New jersey.

[2]. Society, J.Macionis, John, Plummer, Ken, third edition, 2005, printed By Nlateu Cromo Art Grafics, Spain.

[3]. Sociology, T.Schaefer,Richard and.Lamm,Robert, fourth edition, 1992. Printed in -United States of America

[4]. www.Wikipedia.com. About ADHD definition and history. 